\title{
Effets de supplémentation des feuilles séchées de Calotropis procera (WILLD) R. Br. sur les performances de croissance et la charge parasitaire des ovins en saison pluvieuse au Burkina Faso
}

\author{
Peingued-Ouindé Oumarou KANAZOE ${ }^{1,2}$, Géneviève $\mathrm{ZABRE}^{2}$, Adama KABORE ${ }^{2 *}$, \\ Almamy KONATE ${ }^{2}$, Amadou TRAORE ${ }^{2}$, H. Hamidou TAMBOURA ${ }^{2}$, \\ A. M. Gaston BELEM ${ }^{3}$, Jeanne MILLOGO ${ }^{1}$ et Boukari LEGMA ${ }^{1}$ \\ ${ }^{1}$ Université Thomas d'Aquin, Faculté des Sciences Techniques, 03 BP 7026 Ouagadougou 03 Burkina Faso. \\ ${ }^{2}$ Département Productions Animales, Institut de l'Environnement et de Recherches Agricoles, \\ 04 BP 8645 Ouagadougou 04, Burkina Faso. \\ ${ }^{3}$ Institut du Développement Rural, Université Polytechnique de Bobo-Dioulasso, \\ 01 BP 3770 Ouagadougou 01, Burkina Faso. \\ *Auteur correspondant; E-mail : ade1_bf@yahoo.fr, Tel : +22670281098
}

\section{RESUME}

L'étude vise à rechercher une solution alternative à la lutte contre les nématodes parasites du tube digestif des ovins en station. Pour cela, deux niveaux de supplémentation avec les feuilles séchées de Calotropis procera sur les performances de croissance et la charge parasitaire ont été étudiés en infestation naturelle. Ainsi, 3 lots homogènes de 5 ovins de race Mossi composés d'un témoin (lot A) exploitant uniquement le pâturage naturel et de deux lots B et C supplémentés respectivement avec 100 et $200 \mathrm{~g}$ de feuilles séchées de $C$. procera en plus du pâturage naturel ont été constitués. Les résultats obtenus révèlent que le fourrage distribué de $C$. procera est riche en matières azotées totales $(19,07 \%$ de MS) avec un taux moyen de cellulose $(23,44 \%$ de MS). Les GMQ et les niveaux d'excrétion des œufs n'ont pas relevé de différence significative $(\mathrm{P}>0,05)$ entre les trois lots avec la supplémentation. Par contre, la supplémentation a entraîné une baisse importante de H. contortus, T. colubriformis et Cooperia spp dans les fèces des lots supplémentés comparés au lot témoin. L'utilisation de $C$. procera pourrait donc être envisagée dans une stratégie de lutte ciblée contre ces parasites, notamment $H$. contortus.

(C) 2017 International Formulae Group. All rights reserved.

Mots clés: Calotropis procera, supplémentation, GMQ, OPG, ovins.

\section{Supplementation effects of Calotropis procera dried leaves on growth performance and strongyle infection of sheep in the rainy season in Burkina Faso}

\begin{abstract}
The study aims at finding an alternative solution to the control of parasitic nematodes of sheep digestive tract in the station. For this, two levels of supplementation with Calotropis procera dried leaves on the growth performances and strongyle infestation were studied in natural infestation. Thus, three
\end{abstract}


homogeneous groups of 5 sheep of Mossi breed composed of control group (lot A) exploiting only the natural grazing and two groups B and $\mathrm{C}$ supplemented respectively with 100 and $200 \mathrm{~g}$ of C. procera dried leaves in addition to the pasture have been composed. Obtained results reveal that the distributed fodder of $C$. procera is rich in total nitrogenous matter $(19.07 \% \mathrm{DM})$ with average cellulose content $(23.44 \% \mathrm{DM})$. The GMQs and egg excretion levels did not show a significant difference $(\mathrm{P}>0.05)$ between the three groups with supplementation. On the other hand, it induced a significant decrease of Haemonchus contortus, Trichostrongylus colubriformis and Cooperia spp in the fecal matter of the supplemented groups compared with the control group. The use of $C$. procera could therefore be considered in a targeted control strategy against these parasites, especially $H$. contortus.

(C) 2017 International Formulae Group. All rights reserved.

Keywords: Calotropis procera, supplementation, ADG, EPG, sheep.

\section{INTRODUCTION}

Pays sahélo soudanien, le Burkina Faso est l'un des pays d'Afrique occidentale où l'activité d'élevage occupe une place socioéconomique majeure. Cet élevage recèle d'énormes potentialités au regard de l'importance et de la diversité du cheptel national en général et de celui des ruminants en particulier qui se compose d'un effectif de 8566448 bovins, 8490513 ovins et 12712705 caprins (MRA, 2011). Cette majorité des petits ruminants (ovins et caprins) s'explique par le fait qu'ils sont élevés par plus de $82 \%$ de la population (MRA, 2004). Ces animaux sont élevés pour répondre à plusieurs fonctions au sein des exploitations familiales rurales. Entre autres, on peut citer l'épargne, les us coutumiers, la fourniture de la viande, du lait cru, la peau, la production du fumier. L'élevage reste ainsi la première source pour $38,8 \%$ des ruraux pour accéder aux services sociaux de bases (Zerbo et Siri, 2012).

Cependant, le secteur de l'élevage demeure confronté à des contraintes de diverses natures parmi lesquelles la dégradation et la rareté des ressources naturelles, la réduction des parcours pastoraux et les maladies. L'élevage en général et celui des petits ruminants en particulier reste confronté à cette situation de déficit alimentaire sur une longue période de l'année (9 mois sur 12) et associé aux maladies parasitaires digestives en période d'abondance de pâturages naturels. En effet, le mode de conduite pratiqué est essentiellement axé sur l'exploitation du pâturage naturel qui a pour conséquence directe les parasitoses gastrointestinales, contrainte majeure de l'élevage des petits ruminants sous les tropiques humides (Krecek et Waller, 2006). Face à ces contraintes d'ordres alimentaire et sanitaire, l'usage des plantes fourragères à vertu thérapeutique se révèle être une alternative à la problématique des parasites gastrointestinaux vu le coût élevé des produits conventionnels et le manque d'agents vétérinaires dans certaines régions du Burkina (Kaboré et al., 2007).

Calotropis procera (WILLD) R. Br. est un arbuste de la famille des Apocynaceae et a pour nom local Poutrepouga en Mooré dans le Plateau Central du Burkina Faso où il est très répandu et reconnu en médecine traditionnelle pour ses effets anthelminthiques (OMS, 2004). C'est dans cette optique que la présente étude se propose d'évaluer les effets nutritionnels et anthelminthiques de cette plante en vue de mieux conseiller ultérieurement les éleveurs ruraux sur son utilisation en élevage des petits ruminants.

\section{MATERIEL ET METHODES}

\section{Site de l'étude}

La présente étude a été réalisée à la station expérimentale de Saria de l'Institut de l'Environnement et de la Recherche Agricole (INERA) implantée dans la zone périphérique de la commune de Koudougou, province du Boulkiémdé. Cette station est située entre $12^{\circ} 16^{\prime}$ Nord de latitude, $2^{\circ} 09^{\prime}$ Ouest de longitude, à $300 \mathrm{~m}$ d'altitude. Le climat du 
site de l'étude est de type Nord soudanien (Guinko, 1984) et caractérisé par deux saisons: une saison pluvieuse qui s'étale de juin à septembre et une saison sèche de sept mois allant d'octobre à mai. Les températures sont généralement élevées au cours de l'année avec toutefois une modération en hivernage $\left(25-35^{\circ} \mathrm{C}\right)$ où l'humidité relative est comprise entre 60 et $80 \%$. La formation végétale de la station est caractérisée par la présence d'une savane à graminées annuelles, à arbres et arbustes. Les ligneux fréquemment rencontrés sont Parkia biglobosa, Buttyrospermunsp, Faidherbia albida, Adansonia digitata et Guiera senegalensis. La strate herbacée est surtout représentée par les espèces comme Pennisetum sp et Andropogon sp, Loudetia togoensis $L$. et Schoenefeldia gracilis (Guinko, 1984).

\section{Matériel végétal}

Les feuilles séchées de C. procera (référence $\mathrm{n}^{\circ} 283 \mathrm{du} 5 / 02 / 1974$ ) ont été récoltées à Ouagadougou et utilisées pour conduire l'expérimentation à la station expérimentale de Saria au Burkina Faso. Pour cela, la récolte des feuilles a été faite en juillet-aout 2015 et séchées pendant 7 jours en moyenne dans un local à l'abri du soleil et de la poussière. Ensuite, le fourrage sec obtenu a été haché finement afin de faciliter leur préhension par les animaux durant l'expérimentation.

\section{Matériel animal}

Quinze ovins de race Mossi, de sexe femelle et non gestantes, âgées de 10 à 18 mois et provenant de la bergerie de la station expérimentale de Saria ont été utilisés pour l'expérimentation in vivo en infestation naturelle durant la saison des pluies d'août à septembre 2015. Ces animaux dont le poids moyen était de $16,4 \pm 2,9 \mathrm{~kg}$ ont été élevés selon le système traditionnel de type extensif prédominant dans le milieu d'étude et répartis en trois lots homogènes (en poids) durant l'expérimentation. Durant la période de l'essai, les besoins alimentaires des animaux sont couverts généralement par les pâturages naturels. Trois semaines avant l'expérimentation, tous les animaux ont été vaccinés contre la pasteurellose et subi un déparasitage interne («Bolumisole 3 » en suivant les recommandations du fabricant) et externe.

\section{Méthodologie de l'étude}

L'expérimentation en infestation naturelle a été conduite sur une période de 28 jours en appliquant le protocole subissant les trois traitements suivants :

- le lot A a exploité uniquement le pâturage naturel (témoin);

- le lot B a exploité le pâturage naturel et reçu $100 \mathrm{~g}$ de feuilles séchées de $C$. procera associés à $2 \%$ de sel brut;

- Le lot $\mathrm{C}$ a exploité le pâturage naturel et reçu $200 \mathrm{~g}$ de feuilles séchées de $C$. procera associés à $2 \%$ de sel brut.

Tous les animaux des trois lots ont été conduits au pâturage tous les jours de $8 \mathrm{~h}$ à 16 $\mathrm{h}$ et les lots $\mathrm{B}$ et $\mathrm{C}$ ont reçu la supplémentation le soir à $17 \mathrm{~h}$ dans leurs loges pendant toute la durée de l'expérimentation. Les refus des lots supplémentés ont été collectés et pesés chaque matin avant de servir le nouveau supplément le soir au retour des pâturages.

Les paramètres mesurés au cours de l'expérimentation ont été :

- les valeurs bromatologiques des feuilles séchées de $C$. procera distribué en supplémentation. Pour cela, un échantillon de 500 g d'un mélange de ces feuilles a été utilisé pour déterminer les composants chimiques qu'il renferme en adoptant la méthode de l'AOAC (1990) pour les matières sèche (MS), minérale (MM), organique (MO) et les azotées totales (MAT) et la méthode de Van Soest et al. (1991) pour les fibres (CB : cellulose brute, NDF : neutral detergent fiber, ADF : acid detergent fiber, ADL : acid detergent lignin);

- la croissance pondérale des animaux des trois lots d'ovins en expérimentation grâce à différentes pesées réalisées aux dates $\mathrm{J}_{0}, \mathrm{~J}_{3}, \mathrm{~J}_{7}$, $\mathrm{J}_{14}, \mathrm{~J}_{21}$ et $\mathrm{J}_{28}$ à l'aide d'un peson de marque 
SAFTER (portée de $50 \mathrm{~kg}$ avec $200 \mathrm{~g}$ de précision);

- le niveau de réduction d'excrétion des œufs des strongles gastro-intestinaux aux dates $\mathrm{J}_{0}$, $J_{3}, J_{7}, J_{14}, J_{21}$ et $J_{28}$. Le nombre d'œufs par gramme de fèces (opg) a été déterminé à chacune de ces périodes en réalisant des coproscopies en suivant la technique de MacMaster préconisée par Hansen et Perry (1994) et des coprocultures conformément à la méthode décrite par Raynaud (1970).

\section{Analyses statistiques}

Les données collectées ont servi à calculer les moyennes des paramètres mesurés avant d'être soumises à une analyse de variance à une voie pour discriminer leurs influences. Le test de Tukey-Kramer à $5 \%$ de signification a été utilisé pour comparer les moyennes obtenues avec le logiciel Costat (version 6.20.4). Pour le paramètre OPG, une transformation par la formule de $\log (\mathrm{x}+1)$ a été préalablement réalisée pour normaliser les données collectées.

\section{RESULTATS}

Valeurs bromatologiques de $C$. procera

Les valeurs bromatologiques des feuilles de $C$. procera sont présentées dans le Tableau 1. Il révèle que les feuilles séchées de C. procera renferment au stade de leur récolte une teneur en matière sèche (MS : 84,65\%) et minérale (MM : 17,02\% MS) élevée, une bonne teneur en matières azotées totales (MAT: 19,07\% MS) et un faible taux de fibres pariétales.

\section{Consommation de $\boldsymbol{C}$. procera}

La distribution des feuilles séchées de C. procera n'a entraîné aucune perturbation au niveau des animaux des lots traités. En effet, la Figure 1 montre que les ovins supplémentés ont ingéré normalement les quantités de feuilles séchées qui leur ont été distribuées durant toute la période de l'étude. Cette consommation a varié de 7,5 $\pm 3,5 \mathrm{~g}$ à $100 \mathrm{~g}$ pour le lot $\mathrm{B}$ et de $13,5 \pm 9,1 \mathrm{~g}$ à $200 \mathrm{~g}$ pour le lot $\mathrm{C}$ respectivement du $1^{\mathrm{er}}$ et au $28^{\text {ème }}$ jour de l'essai.

Le Tableau 2 présente un récapitulatif de l'ingestion moyenne du fourrage et l'équivalent en azote par animal et par jour de $C$. procera. On note une consommation importante des feuilles séchées de la plante durant l'essai au niveau des lots supplémentés. Le taux moyen d'ingestion des feuilles séchées de $C$. procera a été de $92,32 \%$ pour le lot $\mathrm{B}$ et de $68,6 \%$ pour le lot $\mathrm{C}$ avec des quantités d'ingestion qui ont accru avec le temps du début à la fin de l'essai (Figure 1). Entre les deux lots traités, le maximum ingéré a été atteint plus rapidement au niveau du lot $\mathrm{B}\left(3^{\text {ième }}\right.$ jour $)$ que du lot $\mathrm{C}\left(28^{\text {ième }}\right.$ jour $)$. En outre, on observe que le lot $\mathrm{C}$ a ingéré presque le double des quantités de matières sèches et de matières azotées ingérées par les animaux du lot B.

\section{Performance pondérale des animaux}

L'évolution du poids des animaux en fonction des traitements appliqués est rapportée à la Figure 2. On remarque que les animaux des trois lots ont connu un accroissement de poids pendant la majeure partie de l'essai. En effet, leur poids moyen a légèrement baissé à $\mathrm{J} 3$ (perte de poids de 0,11 $\mathrm{kg}$ pour le lot $\mathrm{A}, 0,14 \mathrm{~kg}$ pour lot $\mathrm{B}$ et 0,039 $\mathrm{kg}$ pour le C) avant de croitre ensuite de manière continue jusqu'au $\mathrm{J}_{28}$. Pendant toute cette période de l'étude, aucune différence significative $(\mathrm{P}>0,05)$ n'a été observée entre les trois lots.

Avec un poids moyen de $16,4 \pm 1,4 \mathrm{~kg}$ au début de l'essai, les animaux du lot témoin (lot $\mathrm{A})$ ont eu à la fin de l'essai un poids moyen de 18,6 $\pm 2,4 \mathrm{~kg}$. Pour les animaux des lots supplémentés avec les feuilles séchées de C. procera, les poids moyens sont passés de $16,3 \pm 3,3$ à 18,64 $\pm 3,9 \mathrm{~kg}$ pour ceux du lot $\mathrm{B}$ et de 16,6 64,3 à $18,64 \pm 4,1 \mathrm{~kg}$ pour le lot $\mathrm{C}$. La comparaison des moyennes calculées à chacune des périodes de l'essai n'a pas montré des différences significatives $(\mathrm{P}>0,05)$ durant l'évolution du poids des animaux du lot témoin et celle des animaux des lots 
complémentés aux feuilles séchées de $C$. procera. De même, aucune différence significative $(\mathrm{P}>0,05)$ entre l'évolution du poids des animaux complémentés à $100 \mathrm{~g}$ de feuilles séchées de $C$. procera et celui des animaux supplémentés avec $200 \mathrm{~g}$ de feuilles séchées de C. procera.

Le gain moyen quotidien (GMQ) des animaux en fonction des traitements est présenté dans le Tableau 3. Les GMQ obtenus par les animaux des trois lots expérimentaux sont statistiquement comparables $(\mathrm{P}>0,05)$ bien que le lot $\mathrm{B}$ ait obtenu le meilleur GMQ $(81,42 \pm 24,01 \mathrm{~g})$ comparé au lot témoin $(78,57 \pm 49,74 \mathrm{~g})$ et au lot $\mathrm{C}(70,00 \pm 49,33$ $\mathrm{g})$. Ce résultat laisse entrevoir que la ration de $100 \mathrm{~g} / \mathrm{j}$ de feuilles séchées de C. procera comme supplément serait meilleur à utiliser.

\section{Charge parasitaire des animaux}

Durant l'étude, l'évolution du nombre d'œufs par gramme de fèces (OPG) des animaux de chaque lot est présentée dans le tableau 4. On observe une infestation continue des animaux au niveau des trois lots expérimentaux du $3^{\text {ème }}$ au $28^{\text {ème }}$ jour de l'étude. Le nombre des OPG est relativement faible au niveau de tous les lots du $3^{\text {ème }}$ au $7^{\text {ème }}$ jours puis croit rapidement du $14^{\text {ème }}$ au $28^{\text {ème }}$ jours, surtout au niveau du lot B (2 450 et 33 150) comparé au lot A (2 050 et 19150$)$ et au lot $\mathrm{C}$ (2 100 et 17350$) \mathrm{du} 21^{\text {ème }}$ au $28^{\text {ème }}$ jours. Durant ces périodes, la comparaison des moyennes des OPG excrétés n'a présenté aucune différence significative $(\mathrm{P}>0,05)$ de l'évolution de ce paramètre entre les animaux du lot témoin (A) et ceux des animaux des lots (B et C) complétés avec les feuilles séchées de C. procera.

Les différentes coprocultures réalisées ont permis de révéler la présence de 4 types parasites nematodes pendant cette étude. Ce sont Haemonchus contortus, Trichostrongylus colubriformis, Cooperia spp. et Oesophagostomum columbianum.

Les prévalences de ces différents parasites observés à la date $\mathrm{J}_{3}$ et à la fin de l'essai sont présentées à la Figure 3. Elles révèlent qu'au début de l'essai, seul le parasite H. contortus était présent dans les coprocultures des lots B $(100 \%)$ et C $(100 \%)$. Le lot A hébergeait les parasites $T$. colubriformis $(37,5 \%)$ et Cooperia spp. (25\%) en plus de $H$. contortus $(37,5 \%)$. A la fin de l'essai, le parasite le plus prévalent était $O$. columbianum (plus de $80 \%$ ) qui a été observé dans les coprocultures des trois lots étudiés. Ensuite vient $H$. contortus avec une prévalence plus élevée au niveau du lot témoin $(14,3 \%)$ qu'au niveau des lots complémentés avec le fourrage de $C$. procera $(0,5 \%$ avec $100 \mathrm{~g}$ et $1,7 \%$ avec $200 \mathrm{~g})$. Quant aux parasites Cooperia spp., ils étaient présents avec une prévalence moindre dans le lot $\mathrm{A}(3,2 \%)$ et le lot B $(0,5 \%)$. Seuls les animaux du lot témoin ont hébergé particulièrement le parasite $T$. colubriformis $(1,6 \%)$.

Tableau 1: Valeurs bromatologiques du fourrage à base de feuilles séchées de C. procera distribuées aux animaux durant l'expérimentation.

\begin{tabular}{|c|c|c|c|c|c|c|c|}
\hline \multirow[t]{2}{*}{ Echantillon } & \multirow{2}{*}{$\begin{array}{l}\text { MS } \\
(\%)\end{array}$} & \multicolumn{6}{|c|}{$\% \mathrm{MS}$} \\
\hline & & MM & MAT & CB & NDF & ADF & ADL \\
\hline C. procera & 84,65 & 02 & 19,07 & 23,44 & 33,14 & 17,10 & 4,89 \\
\hline
\end{tabular}

MS : Matière sèche; MM : Matière Minérale; MAT : Matière Azotée Totale; CB : Cellulose Brute ; NDF : Neutral de Detergent Fiber ; ADF : Acid Detergent Fiber; ADL : Acid Detergent Lignin. 


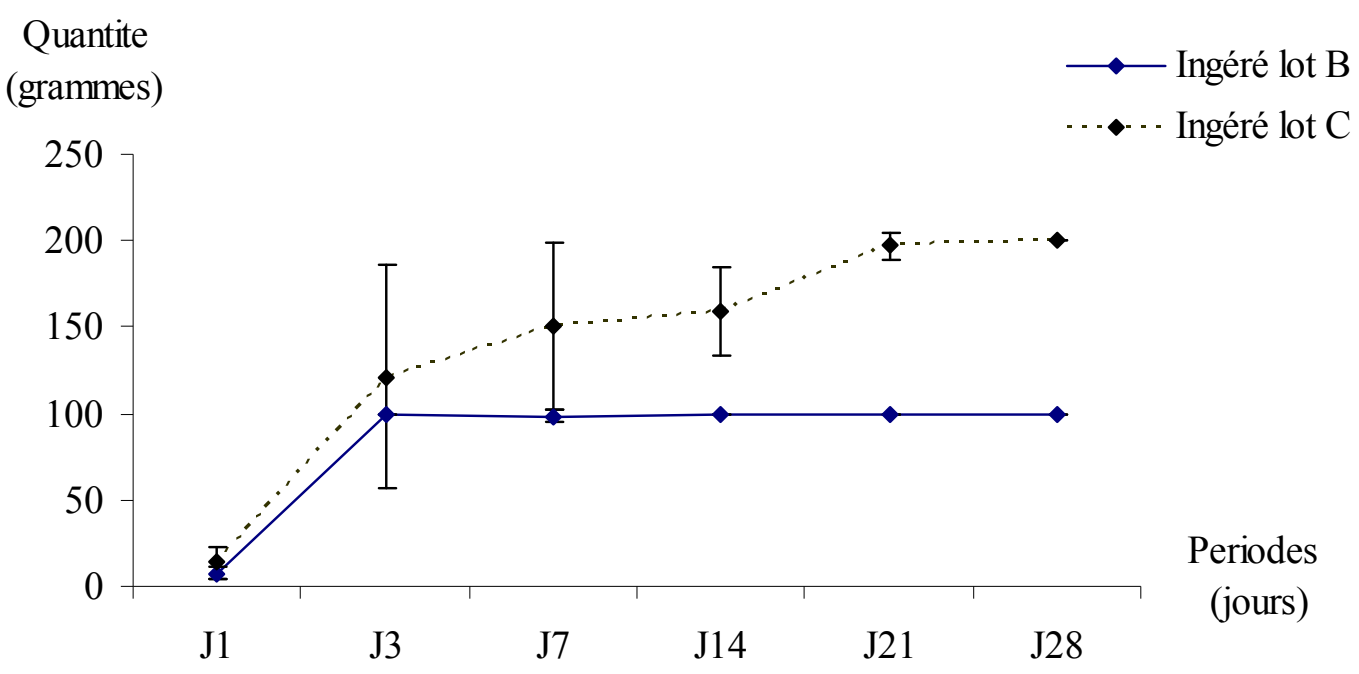

Figure 1 : Evolution de l'ingestion du supplément dans les deux lots traités en fonction du temps.

Tableau 2: Ingestions moyennes du fourrage de C. procera durant l'expérimentation.

\begin{tabular}{|c|c|c|c|c|c|c|}
\hline \multirow{2}{*}{$\begin{array}{c}\text { Quantité } \\
\text { offerte }\end{array}$} & \multicolumn{3}{|c|}{ Quantité totale (g) } & \multicolumn{2}{|c|}{$\begin{array}{l}\text { Quantité par jour } \\
\text { (g) }\end{array}$} & \multirow{2}{*}{$\begin{array}{c}\text { Taux } \\
\text { d'ingestion } \\
\text { moyen }\end{array}$} \\
\hline & Servie & Ingérée & Eq. MS ing. & Eq. MS & Eq. $N$ & \\
\hline Lot B & 2800 & 2585 & 2188,2 & 78,1 & 2,8 & 92,32 \\
\hline Lot $\mathrm{C}$ & 5600 & 3842 & 3252,2 & 116,15 & 4,1 & 68,6 \\
\hline
\end{tabular}

Eq. MS ing. : Equivalent en matière sèche ingérée ; Eq. N: Equivalent en azote

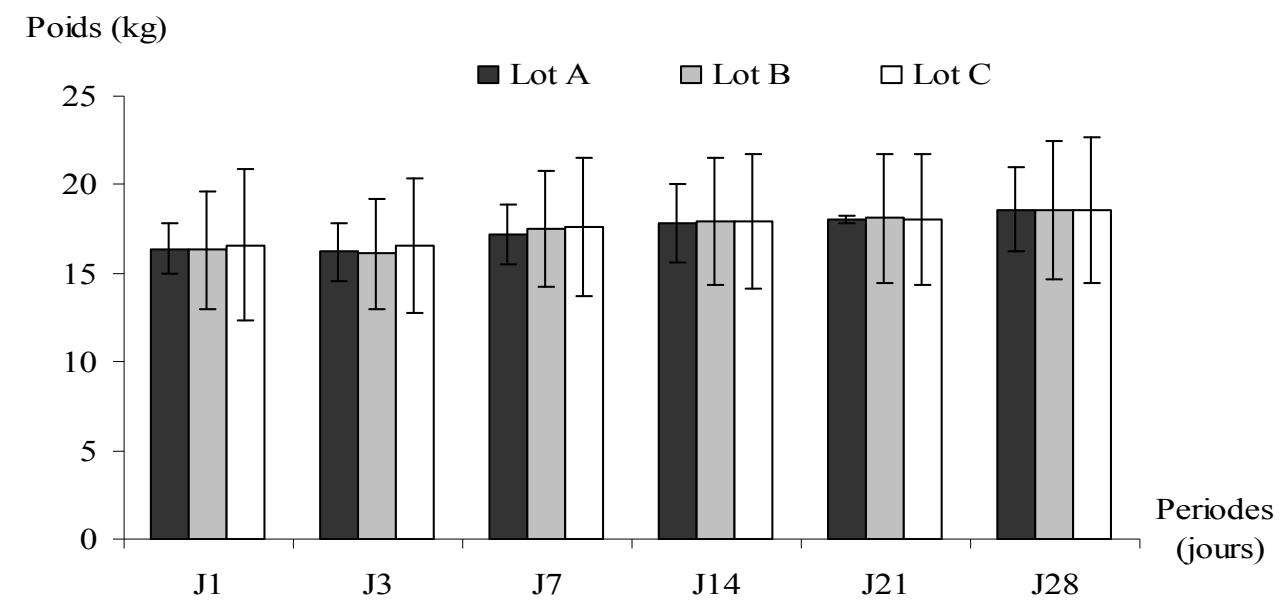

Figure 2 : Evolution des poids moyens des animaux des trois lots expérimentaux durant l'essai. 
Tableau 3: Evolution des gains moyens quotidiens (GMQ) des animaux en fonction des traitements appliqués.

\begin{tabular}{crccc}
\hline Traitements & \multicolumn{2}{c}{ Poids (kg) } & $\begin{array}{c}\text { Gain moyen } \\
(\mathbf{k g})\end{array}$ & $\begin{array}{c}\text { GMQ } \\
\text { (g) }\end{array}$ \\
\cline { 2 - 3 } & Initial & Final & & \\
\hline Lot A & $16,4 \pm 1,4^{\mathrm{a}}$ & $18,6 \pm 2,4^{\mathrm{a}}$ & $2,200 \pm 1,392^{\mathrm{a}}$ & $78,57 \pm 49,74^{\mathrm{a}}$ \\
Lot B & $16,3 \pm 3,3^{\mathrm{a}}$ & $18,64 \pm 3,9^{\mathrm{a}}$ & $2,280 \pm 0,672^{\mathrm{a}}$ & $81,42 \pm 24,01^{\mathrm{a}}$ \\
Lot C & $16,6 \pm 4,3^{\mathrm{a}}$ & $18,64 \pm 4,1^{\mathrm{a}}$ & $1,960 \pm 1,381^{\mathrm{a}}$ & $70,00 \pm 49,33^{\mathrm{a}}$ \\
\hline
\end{tabular}

$(\mathrm{a}, \mathrm{b})$ : Les moyennes portant la même lettre dans la même colonne ne sont pas statistiquement différentes à $\mathrm{P}>0,05$

Tableau 4: Evolution des OPG en fonction des traitements appliqués et du temps.

\begin{tabular}{ccccccc}
\hline \multirow{2}{*}{ Traitements } & \multicolumn{5}{c}{ Périodes (en jours) } \\
\cline { 2 - 7 } & $\mathbf{J 0}$ & $\mathbf{J 3}$ & $\mathbf{J 7}$ & $\mathbf{J 1 4}$ & $\mathbf{J 2 1}$ & $\mathbf{J 2 8}$ \\
\hline Lot A (témoin) & 0 & $60 \pm 41,8$ & $200 \pm 70,7$ & $410 \pm 178,1$ & $2400 \pm 1498,4$ & $3830 \pm 1961,3$ \\
& & $(300)$ & $(1000)$ & $(2050)$ & $(12000)$ & $(19150)$ \\
\hline Lot B $(100 \mathrm{~g} / \mathrm{j})$ & 0 & $110 \pm 108,3$ & $100 \pm 93,5$ & $490 \pm 315$ & $2790 \pm 1491,3$ & $6630 \pm 5179,5$ \\
& & $(550)$ & $(500)$ & $(2450)$ & $(13950)$ & $(33150)$ \\
\hline Lot C $(200 \mathrm{~g} /)$ & 0 & $220 \pm 103,6$ & $180 \pm 57$ & $420 \pm 192,3$ & $2440 \pm 1407$ & $3470 \pm 2877,4$ \\
& & $(1100)$ & $(900)$ & $(2100)$ & $(12200)$ & $(17350)$ \\
\hline
\end{tabular}

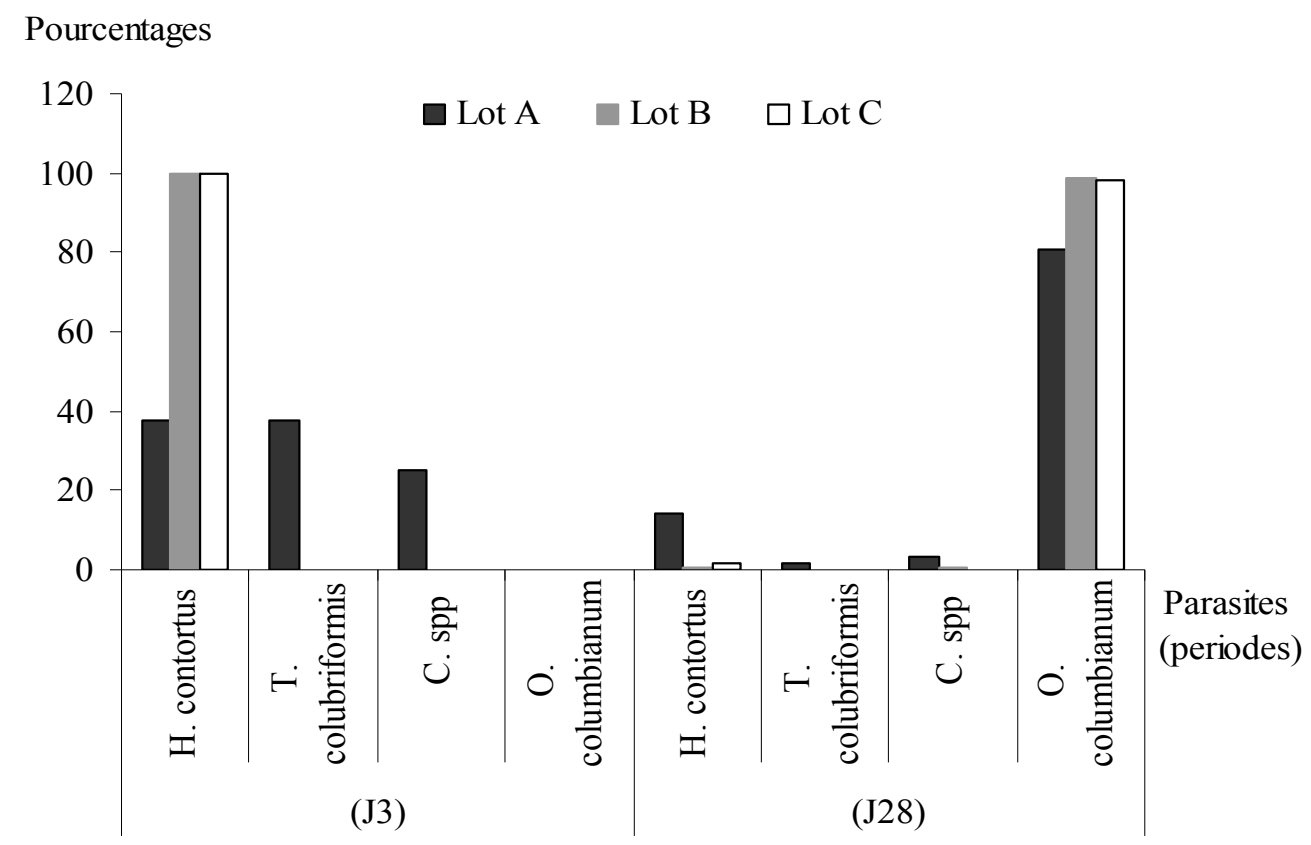

Figure 3 : Prévalence des différents types de parasites observés au début et à la fin de l'essai. 


\section{DISCUSSION}

L'analyse de la valeur alimentaire des feuilles séchées de $C$. procera comme fourrage révèle qu'elle renferme des teneurs élevées en protéines $(19,07 \%)$ et en matières minérales $(17,02 \%)$ et une teneur faible en parois cellulosiques (CB : $23,44 \% \mathrm{MS}$ et NDF : $33,14 \% \mathrm{MS})$. Les valeurs en protéines de l'étude sont supérieures à celles trouvées par Fall (1989) au Sénégal (100 g/kg MS), par contre les valeurs en cellulose brute sont similaires. Au regard de ces résultats, une utilisation judicieuse des feuilles séchées de C. procera dans l'alimentation des petits ruminants pourrait donc être envisagée pour couvrir les besoins azotés de ces animaux. Cette observation est motivée par deux faits. Premièrement, le milieu d'étude est dominé par des sols ferrugineux tropicaux lessivés ou appauvris sur près de $90 \%$ de la superficie totale de la région (MAHRH, 2008). Deuxièmement, les teneurs en protéines du fourrage de $C$. procera étudié sont suffisantes pour induire un bon fonctionnement des micro-organismes du rumen en vue d'accroître les productions, surtout pendant la saison sèche qui est caractérisée par une rareté des ressources fourragères de bonne qualité (Gnanda, 2008). A cela, il faut ajouter que les ressources fourragères naturelles ou cultivées disponibles durant cette période de saison sèche sont constituées essentiellement de fibres composées de cellulose et d'hémicellulose qui sont des sources d'énergie pour les ruminants (Makkar, 2002). Ces sources d'énergie pourraient être mieux exploitées à partir d'une supplémentation stratégique avec les feuilles de $C$. procera dans l'alimentation des petits ruminants dans la région. Dans cette optique, il est impérieux d'explorer des options de complémentation des petits ruminants avec les feuilles séchées de $C$. procera, en association avec les résidus de récolte en vue de valoriser la plante car elle se présente comme une plante largement répandue dans au Burkina Faso.

Dans cette étude, les animaux supplémentés avec les feuilles séchées de $C$. procera se sont comportés de différentes manières en fonction de la quantité de la supplémentation distribuée. En effet, ils ont réalisé un gain de poids pendant l'expérimentation mais c'est seulement le lot $\mathrm{B}$ ayant été supplémenté à hauteur de $100 \mathrm{~g}$ par jour qui a présenté un gain supérieur au lot A (témoin). Cette faible quantité journalière de $100 \mathrm{~g}$ de $C$. procera a entraîné un meilleur taux d'absorption $(92,32 \%)$ de l'aliment comparé au lot ayant reçu $200 \mathrm{~g} \mathrm{du}$ fourrage par jour $(68,6 \%)$ et qui aurait ainsi consommé tout de même une quantité importante de la plante $(116,15$ contre $78,1 \mathrm{~g}$ Eq Ms). Cela permet de penser comme Abbassi et al. (2004) que la perte de poids observée est due aux effets des métabolites secondaires contenus dans la plante, notamment les alcaloïdes qui renfermeraient des effets anti-appétants, à l'origine des pertes de poids que ces auteurs ont observé chez Schistocerca gregaria dans leur étude. En effet, les feuilles séchées de C. procera distribuées aux animaux ont été récoltées en pleine phase végétative, période durant laquelle elles sont riches en substances alcaloïdes dont les précurseurs sont des histamines (Smith, 1980). On pourrait penser que le séchage réalisé dans l'étude a réduit ces effets à condition que les quantités distribuées ne soient pas élevées. Ce qui expliquerait la prise de poids présentée par les animaux du lot $\mathrm{B}$ qui ont consommé $100 \mathrm{~g}$ de feuilles séchées de $C$. procera par jour pendant toute la durée de l'expérimentation. Cette prise de poids est probablement la conséquence du niveau optimum de protéine capable d'entraîner une meilleure digestion ruminale à même d'augmenter la consommation et l'utilisation des fourrages du pâturage naturel en saison des pluies. Dans tous les cas, les résultats des GMQ des lots supplémentés avec les feuilles séchées de $C$. procera sont proches de ceux observés par Gnanda et al. (2015) ((72 à $79 \mathrm{~g} / \mathrm{j})$ sur des ovins nourris avec une ration améliorée à base de gousses de Piliostigma reticulatum en saison sèche. Ils sont également dans les fourchettes trouvées 
par Zoundi et al. (2005) (53,71 à 91,90 g) avec des animaux alimentés avec des rations incorporant 30 à $60 \%$ de concentré « Kibsa » durant la saison sèche également. Toutefois, ils sont supérieurs à ceux obtenus par Boukila et al. (2011) $(32,75 \pm 58,15 \mathrm{~g})$ sur les chèvres naines de Guinée supplémentées avec des feuilles fraîches de manioc (Manihot esculenta) au Cameroun. Ces variations de gain moyen quotidien dans ces différentes études seraient probablement liées aux conditions d'élevage, à l'espèce et à la race.

Dans cette étude, la faune des strongles observée est composée essentiellement de $H$. contortus, T. colubriformis, Cooperia spp. et O. columbianum. Des observations similaires ont été effectuées sur les ovins et les caprins par Belem et al. (2005a; 2005b) au Burkina Faso, et sur les chèvres naines de Guinée au Cameroun par Boukila et al. (2011). Durant l'étude, un accroissement des OPG des strongles a été observé au cours du temps chez les animaux des trois lots. Le lot complémenté avec $100 \mathrm{~g}$ de feuilles de C. procera (lot B : 33 150) a excrété plus d'œufs que ceux des autres lots (lot $\mathrm{A}: 19150$ et lot $\mathrm{C}: 17$ 350) à la fin de l'étude. L'analyse de la faune des strongles montre que l'accroissement des OPG est le fait de la présence des parasites $O$. columbianum au niveau des complémentés alors que dans le lot témoin les quatre types de parasites trouvés sont incriminés. Ce constat révèle alors que les feuilles de $C$. procera présentent un effet anthelminthique ciblé sur les parasites $H$. contortus, $T$. colubriformis et Cooperia spp. du fait de leur faible présence dans les lots complémentés à la fin de l'étude. Ces résultats rejoignent ainsi les travaux de Gupta et al. (2012) qui rapportent que l'administration orale du latex de $C$. procera induit une réduction de l'excrétion des œufs et du nombre de vers adultes de $H$. contortus dans la caillette des ovins. De même, Sidi et al. (2015) qui ont noté dans leur étude que la consommation de la poudre des feuilles de Newbouldia laevis et de Zanthoxylum zanthoxyloïdes perturbent la prolificité de $H$. contortus et de $T$. colubriformis chez les des agneaux Djallonké. Cela prouve que les feuilles de de $C$. procera de l'étude renfermerait des métabolites secondaires à effet anthelminthique. En effet, les travaux de caractérisation phytochimiques de la plante ont relevé la présence de plusieurs phyto-constituants. Il s'agit des hydrocarbures, des protéines, des coumarines, des alcaloïdes, des triterpènes, des saponines, des flavonoïdes, la calotropine, la calotropagénine, la calotoxine, la calotropagénine, les stéroïdes, le stigmastérol et le $\beta$-sitostérol (Parihar et, Balekar, 2016) et des tanins (1,164 g\%) (Sheth, 2011) qui connaissent des activités antimicrobiennes. On peut alors penser que l'activité anthelminthique observée dans l'étude serait due aux actions individuelles ou synergétiques de ces substances secondaires qui agiraient directement ou indirectement sur les parasites rencontrés. Par effet direct, ces métabolites secondaires interviendraient directement sur les vers adultes en agissant sur l'intégrité de leur cuticule (Athanasiadou et al., 2000) ou en perturbant leur fonction de nutrition et de reproduction (Athanasiadou et al., 2001), réduisant ainsi leur fécondité (Paolini et al., 2003). Quant à l'effet indirect, il se manifeste par une stimulation de la réponse immunitaire de l'hôte. Les tanins, notamment les tannins condensés contenus dans les feuilles de $C$. procera favoriseraient ainsi une augmentation du flux de protéines assimilables et d'acides aminés au niveau intestinal en protégeant les protéines alimentaires des dégradations ruminales. Cela se traduit généralement par une prise de poids de l'animal infesté comme observé dans l'étude avec les lots supplémentés. Dans le contexte nord soudanien du pays où le parasitisme des petits ruminants au pâturage pourrait engendrer des pertes de production de 15 à plus de 50\% par morbidité et mortalité (Mandonnet et al., 2005), l'utilisation de $C$. procera peut être envisagée pour maintenir leur productivité en milieu rural (Athanasiadou et Kyriazakis, 2004). En effet, le fourrage de la plante est riche en matière azotée et par conséquent, il 
est indiqué pour minimiser les effets physiopathologiques induits par les parasites gastro-intestinaux chez l'animal infesté (Van Houtert et Sykes, 1996). En outre, cette conviction est renforcée par le fait que la consommation de feuilles séchées de $C$. procera au niveau du lot traité n'a pas entraîné de mortalité chez les animaux en expérimentation.

\section{Conclusion}

Les résultats de cette étude en infestation naturelle révèle une réduction ciblée de $H$. contortus, $T$. colubriformis et $C$. spp., parasites d'importance majeure en parasitologie animale chez les ovins exploitant le pâturage naturel et le fourrage séché de $C$. procera. En plus, la complémentation réalisée durant l'étude induit un gain de poids chez l'animal en période de forte infestation parasitaire des pâturages naturels. De ce constat donc, l'utilisation des feuilles de la plante en phytothérapie vétérinaire en milieu rural burkinabé contre les parasites digestifs des petits ruminants, notamment $H$. contortus, T. colubriformis et C. spp. pourrait être justifiée. Elle se présenterait comme une solution alternative au contrôle des parasites et d'alimentation des animaux. Toutefois, il est nécessaire de compléter la démarche analytique en réalisant des études phytochimiques. Cela permettra de mieux préciser les constituants chimiques que la plante renferme dans le milieu et de mieux préciser les conditions optimales de son emploi en élevage des petits ruminants en toute saison.

\section{CONFLIT D'INTERETS}

Les auteurs déclarent qu'il n'y a aucun conflit d'intérêts.

\section{CONTRIBUTIONS DES AUTEURS}

POOK et GZ ont exécuté les travaux et collecté les données ; AKa était l'investigateur principal et a rédigé le manuscrit ; $\mathrm{AKa}, \mathrm{AK}$ et AT ont supervisé les travaux ; HHT,
AMGB, JM et BL ont lu la version finale du manuscrit.

\section{REMERCIEMENTS}

Les auteurs remercient l'Agence Internationale de l'Energie Atomique (AIEA) pour son soutien en réactifs et équipements au Laboratoire de Biologie et de Santé Animale (LaBioSA) de l'INERA.

\section{REFERENCES}

Abbassi K, Atay KadiriI Z, Ghaout S. 2004. Biologie activity of Calotropis procera (Ait. R. Br) leaves on the desert locust (Schistocerca gregaria, Forsk. 1775). Zool. Baetica, 15: 153-166.

AOAC (Association of Official Analytical Chemist). 1990. Official Method of Analysis (15 ${ }^{\text {th }}$ edn). AOAC : Washington DC.

Athanasiadou S, Kyriazakis I, Jackson F, Coop RL. 2001. Direct anthelmintic effects of condensed tannins towards different gastrointestinal nematodes of sheep: in vitro and in vivo studies. Vet. Parasitol., $\quad$ 99(3): 205-219. https://doi.org/10.1016/S03044017(01)00467-8

Athanasiadou S, Kyriazakis I, Jackson I, Brandt K. 2000. Determination of the most appropriate in vitro test assess the efficacy of substances extracted from bioactive forages. Vet. Record., 146: 728-732.

Athanasiadou S, Kyriazakis I. 2004. Plant secondary metabolites: Antiparasitic effects and their role in ruminant production systems. Proc. Nut. Soc., 63: 631-639.

DOI: $10.1079 /$ PNS2004396

Belem AMG, Kaboré A, Bessin R. 2005a. Gastrointestinal helminthes of sheep in the central, eastern and northern parts of Burkina Faso. Bulletin of Animal Health and Productions in Africa, 53: 13-23.

Belem AMG, Kaboré A, Bessin R. 2005b. Variations saisonnières des helminthes gastro-intestinaux chez la chèvre du 
plateau central du Burkina Faso. Revue d'Elevage et de Médecine Vétérinaire en Pays Tropicaux, 58(1-2): 37-43.

Boukila B, Tendonkeng F, Lemoufouet J, Pamo ET. 2011. Effets de différents niveaux de supplémentation des feuilles fraîches de manioc (Manihot esculenta) sur les performances de croissance, la charge parasitaire et quelques caractéristiques du sang chez la chèvre naine de Guinée. Bulletin of Animal Health and Productions in Africa, 59: 345-356.

Fall ST. 1989. Utilisation d'Acacia albida et de Calotropis procera pour Améliorer les Rations des Petits Ruminants au Sénégal. The Use of Acacia albida and Calotropis procera for Improving Small Ruminant Diets in Senegal, Wilson RT Azeb M (eds). 1989. African small ruminant research and development. ILCA: Addis Ababa, Ethiopia.

Gnanda BI. 2008. Importance socioéconomique de la chèvre du Sahel burkinabé et amélioration de sa productivité par l'alimentation. Thèse de Doctorat Unique en Développement Rural, option Système de Production Animale, Spécialité Nutrition et alimentation, Institut du développement rural (IDR), Université polytechnique de Bobo- Dioulasso (UPB), Burkina Faso. 188pages+annexes.

Gnanda BI, Nignan $\mathrm{M}$, Ouédraogo S, Wereme N'Diaye A, Traoré O, Sinon B. 2015. Influence d'une co-construction de rationnement amélioré sur les performances d'embouche ovine paysanne dans la commune rurale de Korsimoro au Burkina Faso. International Journal of Biological and Chemical Sciences, 9(3): 1544-1556. DOI : http://dx.doi.org/10.4314/ijbcs. v9i3.35

Guinko S. 1984. Végétation de la HauteVolta. Thèse de Doctorat, Université de Bordeaux III (France), 394 p.
Gupta S, Gupta B, Kapoor K, Sharma P. 2012. Ethnopharmacological potential of Calotropis procera: an overview. International Research Journal of Pharmacy, 3(12): 19-22.

Hansen J, Perry B. 1994. The Epidemiology, Diagnosis and Control of Helminth Parasites of Ruminants ( $2^{\text {nd }}$ édn). ILRAD/ILCA. Addis Abéba; 171.

Kaboré A, Tamboura HH, Belem AMG, Traoré A. 2007. Traitements ethnovétérinaires des parasitoses digestives des petits ruminants dans le plateau central du Burkina Faso. International Journal of Biological and Chemical Sciences, 1(3): 297-304. http://dx. doi.org/10.4314/ijbcs.v1i3.39711

Krecek RC, Waller PJ. 2006. Towards of implementation of the "basket of option" approach to helminth parasite control of livestock: Emphasis on the Tropics/ subtropics. Vet. Parasitol., 139: 270-282. DOI: $10.1016 /$ J.VETPAR.2006.04.018

Makkar HPS. 2002. Application of the in vitro method in the evaluation of feed resources, and enhancement of nutritional value of tannin-rich tree/browse leaves and agro-industrial by-products. In: Development and field evaluation of Animal Feed supplementation packages. Proceeding of the final review meeting of an IAEA Technical Co-operation Regional AFRA Project organized by the Joint FAO/IAEA Division of Nuclear Techniques in Food and Agriculture and held in Cairo, Egypt, 25-29 November 2000. Pp 23-40.

Mandonnet N, Bachand M, Mahieu M, Arquet R, Baudron F, Abinne-Molza L, Varo H, Aumont G. 2005. Impact on productivity of peri-parturient rise in fecal egg counts in Creole goats in the humid tropics. Vet. Parasitol., 134(3-4): 249-259. DOI:10.1016/j.vetpar.2005.07.019

Ministère de l'Agriculture, de l'Hydraulique et des Ressources Halieutiques (MAHRH). 2008. Programme de 
spécialisation

Régionale, Ouagadougou/Burkina Faso, 99 pages. O

Ministère des Ressources Animales (MRA). 2004. Les Statistiques du Secteur de l'Elevage au Burkina Faso. Direction des études et de la planification/Services des statistiques animales : Ouagadougou/Burkina Faso ; 54 p.

Ministère des Ressources Animales (MRA). 2011. Statistiques du sous-secteur de l'élevage. DGPSE. Ouagadougou/ Burkina Faso ; 155 p.

Organisation Mondiale de la Santé (OMS). 2004. Document cadre de politique nationale en matière de médecine et pharmacopée traditionnelles, $18 \mathrm{p}$.

Paolini V, Dorchies P, Hoste H. 2003. Effects of sainfoin hay on gastrointestinal nematode infections in goats. Vet. Rec., 152(19): 600-601. DOI: 10.1136/VR.152.19.600-b

Parihar G, Balekar N. 2016. Calotropis procera: A phytochemical and pharmacological review. Thai Journal of Pharmaceutical Sciences, 40(3): 115131.

Raynaud JP. 1970. Etude de l'efficacité d'une technique de coproscopie quantitative pour le diagnostic de routine et le contrôle des infestations parasitaires des bovins, ovins, équins et porcins. Ann. Parasitol. Hum. Comp., 45, 321-342. PMID: 5531507

Sheth F. 2011. Range of Seasonal phytochemical Variation in Calotropis procera (Ait) R.Br., Int. J. Med. Arom. Plants, 1(2): 180-183.

Sidi IYMS, Azando EVB, Olounlade PA, Hounzangbe-Adote MS. 2015. Effets combinés des feuilles de Newbouldia laevis et de Zanthoxylum zanthoxylö̈des sur les nématodes parasites gastrointestinaux des ovins Djallonké. International Journal of Biological and Chemical Sciences, 9(4): 2078-2090. http://dx.doi.org/10.4314/ijbcs.v9i4.30

Smith TA. 1980. Plant amines. In Encyclopedia of Plant Physiology, Secondary Plant Products (Vol. 8), Bell EA, Charlwood BV (eds). Springer: Berlin-Heidelberg-New York; 433-454.

Van Houtert MFJ, Sykes AR. 1996. Implications of nutrition for the ability of ruminants to withstand gastrointestinal nematode infection. Int. J. Parasitol., 26: 1151-1168. DOI: 10.1016/S00207519(96)00120-8

Van Soest JP, Robertson JB, Lewis BA, 1991. Methods for dietary fibre, neutral detergent fibre and non-starch polysaccharides in relation to animal nutrition. Journal of Dairy Science, 74: 3583-3597. DOI:10.3168/JDS.S00220302 (91)78551-2

Zerbo A, Siri D. 2012. Contribution de l'élevage à l'économie et à la lutte contre la pauvreté, les déterminants de son développement. Ouagadougou/Burkina Faso, 67 p.

Zoundi SJ, Sawadogo L, Nianogo AJ. 2005. Utilisation de blocs multinutritionnels en substitution partielle de concentré pour l'engraissement des ovins au sein des systèmes mixtes agriculture-élevage du Plateau Central du Burkina. Journal des Sciences, 14(4): 15-27. 